UCLA/94/TEP/44

UFIFT-HEP-94-17

\title{
CASIMIR FORCES BETWEEN BEADS ON STRINGS AND MEMBRANES
}

\author{
Eric D'Hoker * \\ Physics Department \\ University of California \\ Los Angeles, CA 90024-1547 \\ Pierre Sikivie ** \\ Physics Department \\ University of Florida \\ Gainesville, FL 32611-8440 \\ and \\ Institute for Theoretical Physics \\ University of California \\ Santa Barbara, CA 93106-4030 \\ Youli Kanev *** \\ Physics Department \\ University of Florida \\ Gainesville, FL 32611-8440
}

\begin{abstract}
We develop a general formalism to calculate the force between beads attached to a flat $d$-dimensional membrane due to the quantum fluctuations of the membrane. The interaction potential is derived as a function of $d$ and the membrane energy density, tension, stiffness and temperature. We find that the induced interactions turn off when $d$ exceeds a certain critical dimension. The potential is attractive in all cases where it is non-zero and at finite temperature falls off exponentially for large distances.
\end{abstract}

\footnotetext{
* E-mail address : dhoker@uclahep.physics.ucla.edu

** E-mail address : sikivie@ufhepa.phys.ufl.edu

*** E-mail address : kanev@phys.ufl.edu
} 
In a previous paper [1], two of us derived the force between two beads on a straight string at rest due to the quantum fluctuations of the string. We obtained the interaction potential by explicitly summing the zero-point energies of all the string modes. In the present paper we generalize our results to the case of beads on 2-dimensional and 3dimensional membranes, to allow for stiffness of the string or membrane, and to allow for finite temperature. The methods we use in this paper are much more efficient than those of Ref. [1]. They yield a set of formulae which can be directly applied to a large class of problems of this type. Related issues have recently been discussed in the papers of ref. [2].

The physical system we consider is a straight string or flat membrane, at rest, to which are attached masses $m_{j}$ at locations $\vec{x}_{j}(j=1,2 \ldots N)$. Let $d$ be the dimension of the membrane and $D$ the dimension of the (flat) space in which the membrane is embedded. For example, for a string in physical space, $d=1$ and $D=3$. The membrane has $D-d$ transverse and $d$ longitudinal directions in which to oscillate. For the sake of simplicity, we will first assume that the beads can slide freely along the membrane. In that case, the longitudinal oscillations of the membrane decouple from the beads and we only have to consider its transverse oscillations when calculating the interaction potential between the beads. We will discuss the contribution from the longitudinal oscillations, in case the beads are stuck at a particular location on the membrane, at the end of the paper.

The action describing the dynamics of the displacement $\varphi(\vec{x}, t)$ of the membrane in any one of its $D-d$ transverse directions is

$$
S=\int d t \int d^{d} x \frac{1}{2}\left[\epsilon\left(\frac{\partial \varphi}{\partial t}\right)^{2}-\tau(\vec{\nabla} \varphi)^{2}-\mu(\vec{\nabla} \cdot \vec{\nabla} \varphi)^{2}+\sum_{j=1}^{N} m_{j}\left(\frac{\partial \varphi}{\partial t}\right)^{2} \delta^{d}\left(\vec{x}-\vec{x}_{j}\right)\right]
$$

where $\epsilon$ is the energy/volume of the membrane, $\tau$ its tension and $\mu$ its stiffness. For the sake of definiteness, we are neglecting at first all higher derivative terms beyond the one due to stiffness. Using standard field theory methods [3], one may derive the following formula for the energy $E$ of the lowest energy state of the membrane with beads attached:

$$
-E=\left.(D-d) \lim _{T_{E} \rightarrow \infty} \frac{1}{2 T_{E}} e^{-\sum_{j=1}^{N} \frac{m_{j}}{2} \int_{-T_{E}}^{T_{E}}} d t\left(\frac{\partial}{\partial t} \frac{\delta}{\delta J\left(\vec{x}_{j}, t\right)}\right)^{2} Z[J]\right|_{\substack{J=0 \\ \text { connected }}}
$$

where

$$
Z[J]=e^{\frac{1}{2} \int d^{d+1} x \int d^{d+1} y J(x) \Delta_{F}(x-y) J(y)}
$$

and

$$
\Delta_{F}(x)=\int \frac{d^{d+1} q}{(2 \pi)^{d+1}} \frac{e^{i q \cdot x}}{\epsilon q_{0}^{2}+\tau \vec{q} \cdot \vec{q}+\mu(\vec{q} \cdot \vec{q})^{2}} .
$$

The only connected graphs that appear on the RHS of Eq. (2) are single loops with an arbitrary number of insertions. The insertions describe the emission/absorption of two virtual $\varphi$ quanta by any one of the $N$ beads. After some algebra, one finds that the RHS 
of Eq. (2) can be expressed in terms of a single integral as follows:

$$
\begin{aligned}
-E & =(D-d) \sum_{p=1}^{\infty} \frac{(-1)^{p}}{2 p} \sum_{j_{1}=1}^{N} \ldots \sum_{j_{p}=1}^{N} m_{j_{1}} \ldots m_{j_{p}} \times \\
& \times \int_{-\infty}^{+\infty} \frac{d w}{2 \pi} w^{2 p} G\left(w,\left|\vec{x}_{j_{1}}-\vec{x}_{j_{2}}\right|\right) G\left(w,\left|\vec{x}_{j_{2}}-\vec{x}_{j_{3}}\right|\right) \ldots G\left(w,\left|\vec{x}_{j_{p}}-\vec{x}_{j_{1}}\right|\right) \\
& =-(D-d) \int_{0}^{\infty} \frac{d w}{2 \pi} \ln \operatorname{det}\left(1+w^{2} M\right),
\end{aligned}
$$

where $M$ is the $N \times N$ matrix of elements $M_{j k}=m_{j} G\left(w,\left|\vec{x}_{j}-\vec{x}_{k}\right|\right)$, and:

$$
G(w,|\vec{x}|)=G(w,|\vec{x}| ; \epsilon, \tau, \mu)=\int \frac{d^{d} q}{(2 \pi)^{d}} \frac{e^{i \vec{q} \cdot \vec{x}}}{\epsilon w^{2}+\tau \vec{q} \cdot \vec{q}+\mu(\vec{q} \cdot \vec{q})^{2}}
$$

The calculation which yields Eq. (5) is similar to that which yields the one-loop effective potential of scalar field theories [4]. In particular, the combinatorial factor $\frac{1}{2 p}$ for a loop with $p$ insertions appears in both cases. It is the product of $\frac{1}{p !}$ from the expansion of the exponential in Eq. (2) and $\frac{1}{2}(p-1)$ ! which is the number of inequivalent cyclical orderings of $p$ objects.

The two-body interaction can be readily obtained from Eq. (5) with $\mathrm{N}=2$ :

$$
\begin{aligned}
V(r) & =E^{N=2}(r)-E^{N=2}(\infty) \\
& =(D-d) \int_{0}^{\infty} \frac{d w}{2 \pi} \ell n\left[1-\frac{m_{1} m_{2} w^{4} G(w, r)^{2}}{\left(1+m_{1} w^{2} G(w, 0)\right)\left(1+m_{2} w^{2} G(w, 0)\right)}\right] .
\end{aligned}
$$

Eq. (7) is far more general and simpler than the corresponding formula in Ref. [1]. The three-body interaction potential $V_{3}\left(\left|\vec{x}_{1}-\vec{x}_{2}\right|,\left|\vec{x}_{2}-\vec{x}_{3}\right|,\left|\vec{x}_{1}-\vec{x}_{3}\right|\right)$ can be similarly obtained from Eq. (5) with $\mathrm{N}=3$, and so on.

For $\mu=0$ (pure tension), we have:

$$
\begin{array}{ll}
G(w, r)=\frac{1}{2 \sqrt{\epsilon \tau} w} e^{-\sqrt{\frac{\epsilon}{\tau}} w r} & \text { for } \mathrm{d}=1 \\
G(w, r)=\frac{1}{2 \pi \tau} K_{0}\left(\sqrt{\frac{\epsilon}{\tau}} w r\right) & \text { for } \mathrm{d}=2 \\
G(w, r)=\frac{1}{4 \pi \tau r} e^{-\sqrt{\frac{\epsilon}{\tau}} w r} & \text { for } \mathrm{d}=3
\end{array}
$$

where $K_{0}(x)$ is the modified Bessel function of the third kind. The $G(w, r)$ for $\mu \neq 0$ can be obtained from those for $\mu=0$ using:

$$
G(w, r ; \epsilon, \tau, \mu)=\frac{1}{\mu\left(q_{2}^{2}-q_{1}^{2}\right)}\left[G\left(q_{1}, r ; 1,1,0\right)-G\left(q_{2}, r ; 1,1,0\right)\right]
$$


where

$$
q_{2}^{2}=\frac{1}{2 \mu}\left[\tau \mp \sqrt{\tau^{2}-4 \epsilon \mu w^{2}}\right]
$$

and $\operatorname{Re} q_{1}$, Re $q_{2}>0$. For the case of pure stiffness $(\tau=0)$, one has:

$$
\begin{aligned}
& G(w, r)=\frac{e^{-\sqrt{\frac{w}{2} \sqrt{\frac{\epsilon}{\mu}}} r}}{2 \sqrt{2 w^{3} \sqrt{\mu \epsilon^{3}}}}\left[\cos \left(\sqrt{\frac{w}{2} \sqrt{\frac{\epsilon}{\mu}} r}\right)+\sin \left(\sqrt{\frac{w}{2} \sqrt{\frac{\epsilon}{\mu}} r}\right)\right] \quad \text { for } \mathrm{d}=1 \\
& G(w, r)=\frac{1}{4 \pi i w \sqrt{\epsilon \mu}}\left[K_{0}\left((1-i) \sqrt{\frac{w}{2} \sqrt{\frac{\epsilon}{\mu}} r}\right)-K_{0}\left((1+i) \sqrt{\frac{w}{2} \sqrt{\frac{\epsilon}{\mu}}} r\right)\right] \quad \text { for } \mathrm{d}=2 \\
& G(w, r)=\frac{e^{-\sqrt{\frac{w}{2} \sqrt{\frac{\epsilon}{\mu}}} r}}{4 \pi r w \sqrt{\epsilon \mu}} \sin \left(\sqrt{\frac{w}{2} \sqrt{\frac{\epsilon}{\mu}} r}\right)
\end{aligned}
$$

Note that $G(w, 0)$ is finite in all cases except when $\mu=0$ and $d=2,3$. In these two cases $G(w, r) \sim(\ell n r), r^{-1}$ respectively as $r \rightarrow 0$. It can be seen from Eq. (7) that $V(r)=0$ when $G(w, 0)=\infty$. In the two cases where $G(w, 0)=\infty$, we introduce a short-distance cut-off $\delta$. In many physical applications such a cut-off is in fact present because of the thickness of the membrane. In particular, a small stiffness acts as a cutoff with $\delta=\sqrt{\mu / \tau}$. The role of the cut-off is to restrict the region of integration in Eq. (6) to $q \lesssim \delta^{-1}$. This in effect replaces $G(w, 0)$ by $G(w, \delta)$.

Using Eqs. (7-11), we have calculated the interaction potential $V_{2}(r)$ in a variety of cases. The results are given in Table I. The numerical coefficients $C_{1} \ldots C_{3}$ that appear there are defined by:

$$
\begin{gathered}
C_{1} \equiv-\int_{0}^{\infty} d y y \ln \left[1-e^{-y}(1+\sin y)\right]=2.34 \\
C_{2} \equiv-4 \int_{0}^{\infty} d y y \ln \left[1-\left(\frac{2}{i \pi}\left[K_{0}((1-i) y)-K_{0}((1+i) y)\right]\right)^{2}\right]=1.84 \\
C_{3} \equiv-\int_{0}^{\infty} d y y \ln \left[1-\frac{4}{y^{2}} e^{-y} \sin ^{2}\left(\frac{y}{2}\right)\right]=0.86 .
\end{gathered}
$$




\begin{tabular}{|c|c|c|c|}
\hline & $\mathrm{d}=1$ & $\mathrm{~d}=2$ & $\mathrm{~d}=3$ \\
\hline $\begin{array}{c}\mu=0 \\
\text { small } \mathrm{m}\end{array}$ & $\frac{-m_{1} m_{2}}{32 \pi \epsilon^{2} r^{3}}\left(\frac{\tau}{\epsilon}\right)^{1 / 2}$ & $\frac{-27 m_{1} m_{2}}{2^{12} \pi \epsilon^{2} r^{5}}\left(\frac{\tau}{\epsilon}\right)^{1 / 2}$ & $\frac{-3 m_{1} m_{2}}{2^{7} \pi^{3} \epsilon^{2} r^{7}}\left(\frac{\tau}{\epsilon}\right)^{1 / 2}$ \\
\hline $\begin{array}{c}\tau=0 \\
\text { small } \mathrm{m}\end{array}$ & $\frac{-3 m_{1} m_{2}}{16 \pi \epsilon^{2} r^{4}}\left(\frac{\mu}{\epsilon}\right)^{1 / 2}$ & $\frac{-m_{1} m_{2}}{4 \pi^{2} \epsilon^{2} r^{6}}\left(\frac{\mu}{\epsilon}\right)^{1 / 2}$ & $\frac{-15 m_{1} m_{2}}{32 \pi^{3} \epsilon^{2} r^{8}}\left(\frac{\mu}{\epsilon}\right)^{1 / 2}$ \\
\hline $\begin{array}{c}\mu=0 \\
\text { large } \mathrm{m}\end{array}$ & $\frac{-\pi}{24 r}\left(\frac{\tau}{\epsilon}\right)^{1 / 2}$ & $\frac{-\pi}{8 r} \frac{1}{(\ell n \delta)^{2}}\left(\frac{\tau}{\epsilon}\right)^{1 / 2}$ & $\frac{-\delta^{2}}{4 \pi r^{3}}\left(\frac{\tau}{\epsilon}\right)^{1 / 2}$ \\
\hline $\begin{array}{c}\tau=0 \\
\text { large } \mathrm{m}\end{array}$ & $\frac{-C_{1}}{2 \pi r^{2}}\left(\frac{\mu}{\epsilon}\right)^{1 / 2}$ & $\frac{-C_{2}}{2 \pi r^{2}}\left(\frac{\mu}{\epsilon}\right)^{1 / 2}$ & $\frac{-C_{3}}{2 \pi r^{2}}\left(\frac{\mu}{\epsilon}\right)^{1 / 2}$ \\
\hline
\end{tabular}

Table I: The contribution to the two-bead interaction potential due to the transverse oscillations of the membrane, in a variety of cases. To obtain $V^{\perp}(r)$, multiply each entry by $\hbar(D-d)$. To obtain the contribution $V^{\|}(r)$ due to longitudinal oscillations, replace $\epsilon$ by $\epsilon-\tau$ and $\left(\frac{\tau}{\epsilon}\right)^{1 / 2}$ by $\left(-\frac{d \tau}{d \epsilon}\right)^{1 / 2}$, and multiply each entry by $\hbar d$.

The cases labeled "small $m$ " in Table I are when $m_{i} w^{2} G(w, 0)<<1(i=1,2)$ inside the integral of Eq. (7) and the interaction potential can be approximated by

$$
V(r)=-(D-d) \frac{m_{1} m_{2}}{2 \pi} \int_{0}^{\infty} d w w^{4} G(w, r)^{2}
$$

The cases labeled "large $m$ " in Table $\mathrm{I}$ is when $m_{i} w^{2} G(w, \delta) \gg 1(i=1,2)$ inside the integral of Eq. (7) and the interaction potential can be approximated by

$$
V(r)=(D-d) \int_{0}^{\infty} \frac{d w}{2 \pi} \ln \left[1-\left(\frac{G(w, r)}{G(w, 0)}\right)^{2}\right]
$$

The critical parameter which distinguishes the small $m$ and large $m$ regimes is $\frac{m}{\epsilon r^{d}}$ in all cases where $G(w, 0)$ is finite. For the two cases where $G(w, 0)$ is infinite, and a shortdistance cut-off $\delta$ was introduced as discussed above, the critical parameter is: $\frac{m}{\epsilon r^{2}} \ln \left(\frac{r}{\delta}\right)$ for $\mu=0, d=2$ and $\frac{m}{\epsilon r^{2} \delta}$ for $\mu=0, d=3$. Note that in these two cases one necessarily enters the large mass regime when $\delta \rightarrow 0$ and that $V(r)$ vanishes in that limit as $(\ln \delta)^{-2}$ and $\delta^{2}$ respectively.

We notice that whenever the potential is non-zero, it is attractive for all values of $r$ and produces an instability under which beads tend to group together in lumps. This type of instability was already noticed for the special case of strings $(d=1)$ in [1].

All the results of Ref. [1] except one (see below) are easily reproduced using Eqs. (7) and (8.1) and the generalization of Eq. (7) for $\mathrm{N}=3$. One statement in Ref. [1] cannot be 
reproduced because it is in error. It concerns the next-to-leading term in $V(r)$ for the case of large masses and $\mathrm{d}=1, \mu=0$. The correct answer is:

$$
V(r)=(D-1)\left(\frac{\tau}{\epsilon}\right)^{1 / 2}\left[-\frac{\pi}{24 r}+\frac{1}{2} \sqrt{\frac{\left(m_{1}+m_{2}\right) \epsilon}{m_{1} m_{2} r}}+\ldots\right] .
$$

Higher order corrections of various sorts may be derived from Eq. (7). For example the expansion of $V(r)$ in small masses to 3rd order for $\mathrm{d}=1, \mu=0$ is:

$$
\begin{aligned}
V(r)= & -(D-1) \frac{1}{32 \pi}\left(\frac{\tau}{\epsilon}\right)^{1 / 2} \frac{m_{1} m_{2}}{\epsilon^{2} r^{3}}\left\{1-\frac{3}{4} \frac{m_{1}+m_{2}}{\epsilon r}\right. \\
& \left.+\left[\frac{3}{4}\left(m_{1}^{2}+m_{2}^{2}\right)+\frac{195}{256} m_{1} m_{2}\right] \frac{1}{\epsilon^{2} r^{2}}+\ldots\right\} .
\end{aligned}
$$

The lowest order correction to the interaction potential in the case of pure tension, $d=1$ and small masses, due to a small but non-zero $\mu$ is:

$$
V(r)=-(D-1) \sqrt{\frac{\tau}{\epsilon}} \frac{m_{1} m_{2}}{32 \pi \epsilon^{2} r^{3}}\left(1+\frac{3 \mu}{2 r^{2}}+\ldots\right) .
$$

Et cetera.

At non-zero temperature $T$, the interaction potential equals the free energy which is given by

$$
F(T)=-\left.T(D-d) e^{-\sum_{j=1}^{N} \frac{m_{j}}{2} \int_{0}^{1 / T} d t\left(\frac{\partial}{\partial t} \frac{\delta}{\delta J\left(\vec{x}_{j}, t\right)}\right)^{2}} W[J]\right|_{\substack{J=0 \\ \text { connected }}} .
$$

In the expression for $W[J]$, Eq. (3), $\Delta_{F}(x)$ must now be replaced by:

$$
\Delta_{T}(x)=\sum_{n=-\infty}^{+\infty} T \int_{-\infty}^{+\infty} \frac{d^{d} q}{(2 \pi)^{d}} \frac{e^{i(\vec{q} \cdot \vec{x}+2 \pi n T t)}}{\epsilon(2 \pi n T)^{2}+\tau \vec{q} \cdot \vec{q}+\mu(\vec{q} \cdot \vec{q})^{2}}
$$

Eq. (5) becomes:

$$
F=(D-d) T \sum_{n=1}^{\infty} \ln \operatorname{det}\left(1+w_{n}^{2} M\right)
$$

with $w_{n}=2 \pi n T$ and $M_{j k}=m_{j} G\left(w_{n},\left|\vec{x}_{j}-\vec{x}_{k}\right|\right)$. Thus, to allow for finite $T$, one simply replaces the integral over $w$ by a sum over the discrete frequencies $w_{n}=2 \pi n T$. In particular:

$$
V(r)=(D-d) T \sum_{n=1}^{\infty} \ln \left[1-\frac{m_{1} m_{2} w_{n}^{4} G\left(w_{n}, r\right)^{2}}{\left(1+m_{1} w_{n}^{2} G\left(w_{n}, 0\right)\right)\left(1+m_{2} w_{n}^{2} G\left(w_{n}, 0\right)\right)}\right] .
$$

For finite $T$ and large $r, G\left(w_{n}, r\right)$ falls off exponentially, as $e^{-2 \pi n r T \sqrt{\frac{\epsilon}{\tau}}}$ if $\mu=0$ and as $e^{-\sqrt{\pi n T \sqrt{\frac{\epsilon}{\mu}}}}$ if $\tau=0$. In that limit the $n=1$ term dominates over all others and

$$
V(r)=-(D-d) T \frac{m_{1} m_{2}(2 \pi T)^{4} G(2 \pi T, r)^{2}}{\left(1+2 \pi m_{1} T G(2 \pi T, 0)\right)\left(1+2 \pi m_{2} T G(2 \pi T, 0)\right)} .
$$


One needs only to substitute the expressions for $G(2 \pi T, r)$, Eqs. (8-11), to obtain the various interaction potentials in that limit. In all cases, the interactions between beads on strings and membranes becomes short ranged as soon as the temperature is non-zero. For small masses and all values of $T$,

$$
V(r)=-(D-d) T m_{1} m_{2} \sum_{n=1}^{\infty} w_{n}^{4} G\left(w_{n}, r\right)^{2} .
$$

In some cases, the sum can be done explicitly; e.g. for $d=1$ and $\mu=0$ :

$$
V(r)=-(D-1) \frac{\pi^{2} m_{1} m_{2} T^{3}}{4 \epsilon \tau} \frac{\cosh \left(2 \pi T r \sqrt{\frac{\epsilon}{\tau}}\right)}{\sinh ^{3}\left(2 \pi T r \sqrt{\frac{\epsilon}{\tau}}\right)} .
$$

For large masses and all values of $T$,

$$
V(r)=(D-d) T \sum_{n=1}^{\infty} \ln \left[1-\left(\frac{G\left(w_{n}, r\right)}{G\left(w_{n}, 0\right)}\right)^{2}\right]
$$

In the case $d=1$ and $\mu=0$, this yields

$$
V(r)=(D-1) T \ln \prod_{n=1}^{\infty}\left(1-\alpha^{n}\right)
$$

where $\alpha=e^{-4 \pi \operatorname{Tr} \sqrt{\frac{\epsilon}{\tau}}}$. This expression is well-known from string theory and dual models and exhibits a duality symmetry under the interchange of $r$ and $1 / r$.

Let us turn to the contribution from longitudinal oscillations of the membrane. Such a contribution exists only if the beads are stuck to the membrane, i.e. they are constrained not only to stay on the membrane but to stay at a particular location on the membrane. In particular, the contribution vanishes in the limit $\tau=\epsilon$, where the membrane becomes invariant under boosts parallel to its surface. The relevant action is:

$$
S=\int d t \int d^{d} x\left\{\frac{\epsilon-\tau}{2}\left[\left(\frac{\partial \varphi}{\partial t}\right)^{2}-v_{L}^{2}(\vec{\nabla} \varphi)^{2}\right]-\mu(\vec{\nabla} \cdot \vec{\nabla} \varphi)^{2}+\sum_{j=1}^{N} \frac{m_{j}}{2}\left(\frac{\partial \varphi}{\partial t}\right)^{2} \delta^{d}\left(\vec{x}-\vec{x}_{j}\right)\right\}
$$

where $\varphi$ is the displacement of the membrane from its equilibrium position in any one of the $d$ longitudinal directions and $v_{L}^{2}=-\frac{d \tau}{d \epsilon}$ is the (velocity) ${ }^{2}$ of longitudinal oscillations. Here we are neglecting all terms with more than two spatial derivatives. The action of Eq. (27) reproduces the equations of motion and stress-energy-momentum tensor of the longitudinal oscillations [5]. Comparison of Eqs. (1) and (27) shows that one can obtain the contribution due to longitudinal oscillations from that due to transverse oscillations by replacing $\epsilon \rightarrow \epsilon-\tau, \tau \rightarrow-(\epsilon-\tau) \frac{d \tau}{d \epsilon}, D-d \rightarrow d$.

Finally, let us point out a generalization in which the oscillations of the $d$-dimensional membrane have a general dispersion law : $\epsilon w^{2}=P(\vec{q} \cdot \vec{q})$, where $P$ is any positive function of $\vec{q} \cdot \vec{q}$ which vanishes when $\vec{q} \rightarrow 0$. When only tension and stiffness terms were 
present in the membrane action, $P$ is a polynomial of second degree in $\vec{q} \cdot \vec{q}$ with vanishing constant term. However, one may imagine many other possible dispersion laws, including arbitrarily high powers of $\vec{q} \cdot \vec{q}$ or even fractional powers of $\vec{q} \cdot \vec{q}$. This may occur when the membrane Lagrangian is viewed as an effective theory resulting from complicated microscopic dynamics. The formalism developed above may be applied to this generalized case in a straightforward way : formulas (5) and (7) are unchanged and in the propagators of (4) and (6), it suffices to replace $\tau \vec{q} \cdot \vec{q}+\mu(\vec{q} \cdot \vec{q})^{2}$ by $P(\vec{q} \cdot \vec{q})$. In particular, we have the Green function

$$
G(w,|\vec{x}|)=\int \frac{d^{d} q}{(2 \pi)^{d}} \frac{e^{i \vec{q} \cdot \vec{x}}}{\epsilon w^{2}+P\left(\vec{q}^{2}\right)} .
$$

We shall examine the behavior of the two-body potential when $P$ approaches the following limits

$$
\begin{aligned}
P(\vec{q} \cdot \vec{q}) & \sim P_{\infty} \times(\vec{q} \cdot \vec{q})^{\alpha_{\infty}} & & \text { for } & & \vec{q} \rightarrow \infty \\
& \sim P_{0} \times(\vec{q} \cdot \vec{q})^{\alpha_{0}} & & \text { for } & & \vec{q} \rightarrow 0
\end{aligned}
$$

When $2 \alpha_{\infty} \leq d$, we have $G(w, 0)=\infty$ and as a result the entire two-body potential vanishes identically. We note therefore that there is a critical dimension above which the fluctuating membrane is unaffected by the extra masses. In particular, as was already noted, the potential vanishes for $d=2,3$ and higher in the case of pure tension. Furthermore, the potential vanishes for $d \geq 4$, if stiffness is the highest derivative term in the membrane action. The presence of a cutoff produces finite but non universal interactions.

If $2 \alpha_{\infty}>d, G(w, 0)$ is finite and we may use the formalism developed above to determine the long and short distance behaviors of the interaction potential corresponding to the limiting behaviors of the dispersion law given in Eq. (29). We find

$$
\begin{array}{llrl}
V(r)=-\frac{m_{1} m_{2}}{2 \pi \epsilon^{2}}\left(\frac{P_{0}}{\epsilon}\right)^{\frac{1}{2}} \frac{1}{r^{2 d+\alpha_{0}}} \int_{0}^{\infty} d u u^{2 d / \alpha_{0}} C\left(\alpha_{0}, u\right)^{2}, & \text { for } \quad r \rightarrow \infty \\
V(r)=-\frac{1}{2 \pi}\left(\frac{P_{\infty}}{\epsilon}\right)^{\frac{1}{2}} \frac{1}{r^{\alpha_{\infty}}} \int_{0}^{\infty} d u \ln \left[1-\frac{C\left(\alpha_{\infty}, u\right)^{2}}{C\left(\alpha_{\infty}, 0\right)^{2}}\right], & \text { for } \quad r \rightarrow 0
\end{array}
$$

The function $C(\alpha, u)$ is given by

$$
C(\alpha, u)=\int \frac{d^{d} p}{(2 \pi)^{d}} \frac{e^{i \vec{p} \cdot \hat{n} u^{1 / \alpha}}}{1+(\vec{p} \cdot \vec{p})^{\alpha}}
$$

In Eq. (31), the function $C(\alpha, u)$ is independent of the arbitrary unit vector $\hat{n}$. This behavior may be verified on the cases that we have already presented in Table I, and are found to be in agreement.

\section{Acknowledgements}

One of us (P.S.) would like to thank Larus Thorlacius and Charles B. Thorn for stimulating discussions. This research was supported in part by NSF grants PHY-9218990, PHY-89-04035 and by DOE contract No. DE-FG05-86ER40272. 


\section{References}

1. E. D'Hoker and P. Sikivie, Phys. Rev. Lett. 71 (1993) 1136.

2. D. Förster, Phys. Lett. 114 A (1986) 115; F. David and S. Leibler, Phys. II France 1 (1991) 959; M. Goulian, R. Bruinsma and P. Pincus, Europhysics Lett. 22 (1993) 145; W. Cai, T.C. Lubensky, P. Nelson and T. Powers, "Measure Factors, Tension and Correlations of Fluid Membranes", UPR-599T preprint (1994)

3. See for example: P. Ramond, "Field Theory: A Modern Primer", Addison-Wesley, 1989.

4. S. Coleman and E. Weinberg, Phys. Rev. D7 (1973) 1888.

5. See for example : J. Hong, J. Kim, and P. Sikivie, Phys. Rev. Lett. 69 (1992) 12611; J. Kim and P. Sikivie, "Wiggly relativistic strings", University of Florida preprint UFIFT-HEP-94-4 (May 1994), to appear in Phys. Rev. D.; B. Carter, "Transonic Elastic Model for Wiggly Goto-Nambu String", hep-th-9411231, CNRS preprint (1994) 